\title{
Global and regional assessment of left ventricle longitudinal strain in patient with non-ST-segment elevation myocardial infarction: going further in left ventricular function in acute settings
}

\section{Sandra Jakšić Jurinjak* \\ Mira Stipčević \\ Boris Starčević \\ Josip Vincelj \\ Jasmina Ćatić \\ Diana Rudan \\ Mario Udovičić}

Dubrava University Hospital Zagreb, Croatia

RECEIVED:

April 15, 2015

ACCEPTED:

April 20, 2015

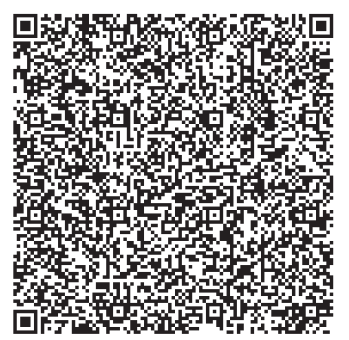

KEYWORDS: left ventricle longitudinal strain, acute coronary syndrome

CITATION: Cardiol Croat. 2015;10(3-4):76-77. | DOI: http://dx.doi.org/10.15836/ccar.2015.76

ORCID: Sandra Jakšić Jurinjak, http://orcid.org/0000-0002-7349-6137 • Mira Stipčević, http://orcid.org/0000-0003-4351-1102 • Boris Starčević, http://orcid.org/0000-0002-3090-2772 • Josip Vincelj, http://orcid.org/0000-0003-0064-9128 • Jasmina Ćatić, http://orcid.org/0000-0001-6582-4201 • Diana Rudan, http://orcid.org/0000-0001-9473-2517• Mario Udovičić, http://orcid.org/0000-0001-9912-2179

*ADDRESS FOR CORRESPONDENCE: Sandra Jakšić Jurinjak, Klinička bolnica Dubrava, Avenija Gojka Šuška 6, HR-10000 Zagreb, Croatia. Phone: +385-1-2902-444. E-mail: sjaksicj@gmail.com

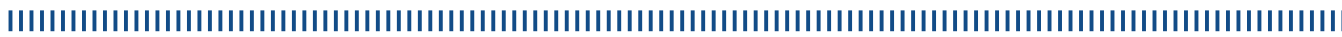

Strain echocardiography is a validated and accurate measure of regional systolic left ventricular function, superior to visual assessment of wall motion in detection and quantification of regional systolic function and is sensitive tool for detection of ischaemial. The ischaemic risk area can only be assessed clinically by perfusion imaging with single photon emission computed tomography or contrast echocardiography, and these modalities are not available in the emergency setting at most hospitals ${ }^{1}$

We demonstrate the use of a non-invasive bedside imaging modality, global and regional assessment of left ventricle (LV) longitudinal strain, to identify acute coronary lesion in the Non-ST-segment elevation (NSTE) acute coronary syndromes (ACS) patient. This has important clinical implications, since acute coronary occlusion is a potentially reversible cause of myocardial ischemia and necrosis, if reperfusion therapy is initiated promptly.

We present an example of a 45-years-old female patient with acute myocardial infarction without ST-segment deviations. The ECG obtained at admission to our hospital showed no evidence of ischemia, and prehospital nonsustained ventricular tachycardia was reported. Troponin I was measured to $10.23 \mu \mathrm{g} / \mathrm{L}$. The patient was stable during 4 hours observation, and had no chest pain after admission. Biplane left ventricular ejection fraction (EF) calculation utilising the Simpson method was performed showing preserved LVEF (52-57\%). However, this method is dependent on visual delineation of the endocardial border, which can be a challenge in patients with poor acoustic conditions. This limitation could contribute to the superiority of global longitudinal strain (GLS) over LVEF, but the same challenges are present when performing two-dimensional speckle tracking ${ }^{2}$. A bull's eye plot of strain values demonstrated a functional risk area of eight adjacent segments with strain greater than or equal to $-12 \%$ (Figure 1, Figure 2). Coronary angiography revealed significant stenosis in the proximal left circumflex artery, the left anterior descending artery, and the right coronary artery with no significant stenosis and no occlusion was found.

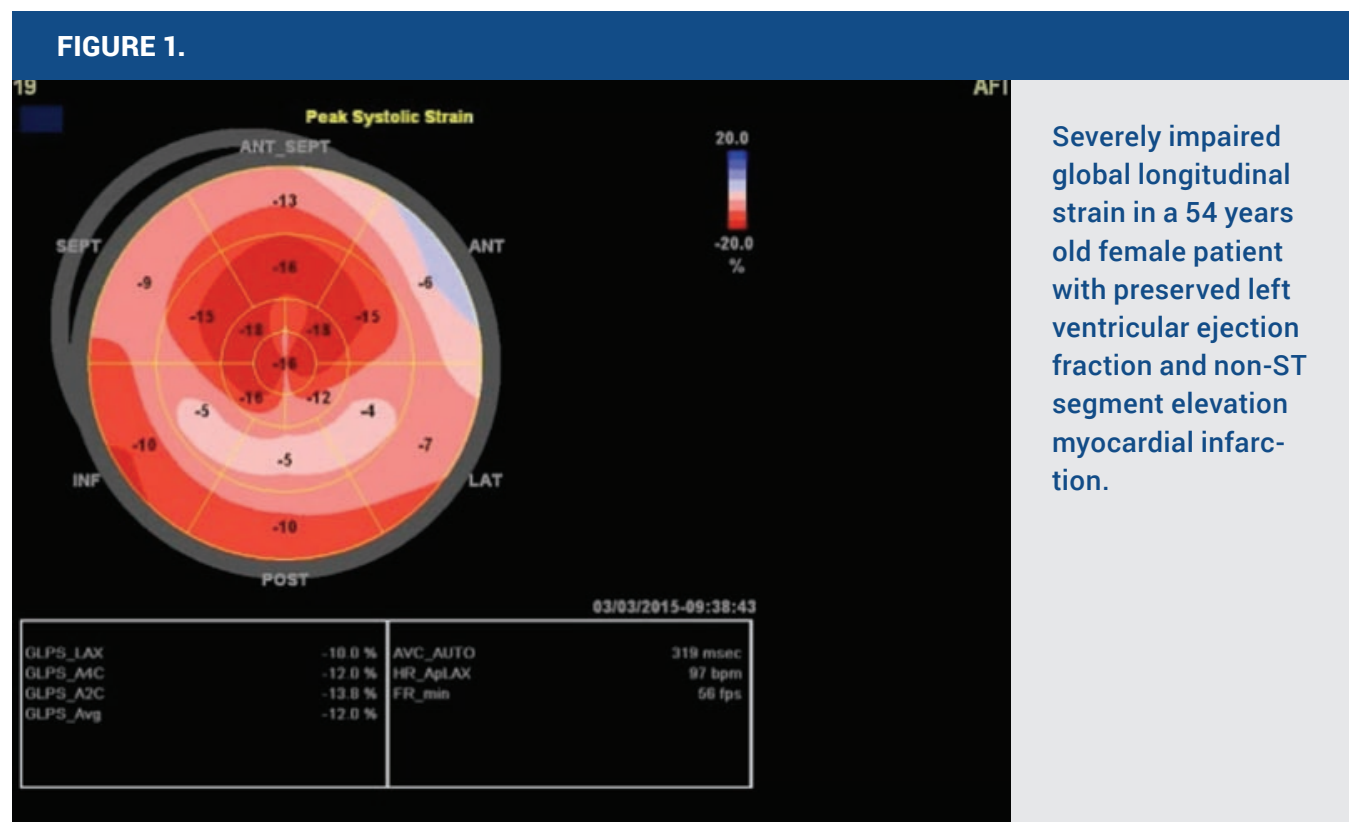


Several mechanisms may explain large areas with dysfunction, most important, transient occlusion with subsequent spontaneous lysis may render the myocardium stunned or necrotic, even if the epicardial vessel is patent when coronary angiography is performed. The prevalence of total occlusion is previously reported to fall during the first $24 \mathrm{~h}$ after infarction ${ }^{1-2}$ indicating that spontaneous lysis is frequent in patients with myocardial infarction. Percutaneous coronary intervention (PCI) was performed at the discretion of the operator, performed by a experienced invasive cardiologist, placing a drug-eluting stent in proximal left circumflex artery.

These patients are also important to identify, as they may profit from intensified antithrombotic therapy and PCI to stabilise the ruptured plaque. The eight-fold increase in mean Tn observed in patients with large risk areas is likely to reflect significantly larger infarctions ${ }^{1,2}$. This may be due to transient occlusion or distal embolisation, and may explain some of the patients presentation

We present 2D strain echocardiography as a diagnostic tool to identify patients with NSTE-ACS patients who may benefit from urgent reperfusion therapy, and can be used in acute setting based on information that can be obtained in the emergency room or invasive centre, to minimise delay Strain echocardiography is valuable tool for better risk stratification and therapy in patients with NSTE-ACS in acute settings.

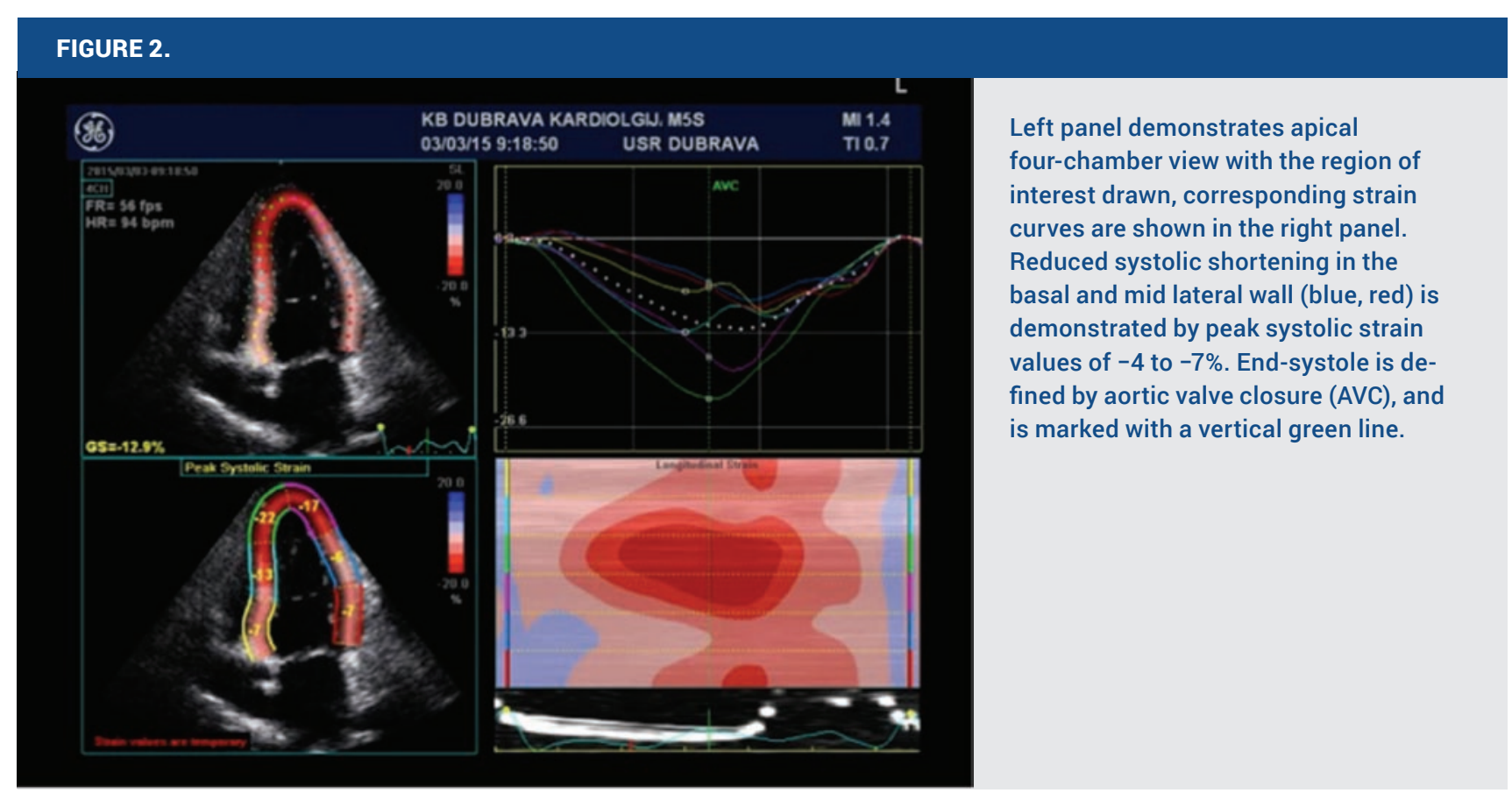

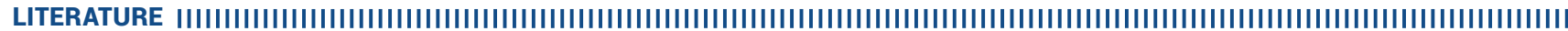

1. Eek C, Grenne B, Brunvand H, Aakhus S, Endresen K, Smiseth OA, et al. Strain echocardiography predicts acute coronary occlusion in patients with non-ST-segment elevation acute coronary syndrome. Eur J Echocardiogr. 2010;116):501-8. DOI: http://dx.doi.org/10.1093/ejechocard/jeq008

2. Ersbøll M, Valeur N, Mogensen UM, Andersen M, Greibe R, Møller JE, et al. Global left ventricular longitudinal strain is closely associated with increased neurohormonal activation after acute myocardial infarction in patients with both reduced and preserved ejection fraction: a two-dimensional speckle tracking study. Eur J Heart Fail. 2012;14(10):1121-9. DOI: http://dx.doi.org/10.1093/eurjhf/hfs107

3. Voigt JU, Pedrizzetti G, Lysyansky P, Marwick TH, Houle H, Baumann R, et al. Definition for common standard for 2D speckle tracking echocardiography: consensus document otthe EACVI/ASE/ Industry Task Force to standardize deformation imaging. Eur Heart J Cardiovasc Imaging. 2015;16(1):1-11. DOI: http://dx.doi.org/10.1093/ehjii/jeu184 